\title{
Cullin 5 regulates Dab1 protein levels and neuron positioning during cortical development
}

\author{
Libing Feng, ${ }^{1,3}$ Nathaniel S. Allen, ${ }^{1,2,3}$ Sergi Simo, ${ }^{1}$ and Jonathan A. Cooper ${ }^{1,2,4}$ \\ ${ }^{1}$ Division of Basic Sciences, Fred Hutchinson Cancer Research Center, Seattle, Washington 98109, USA; ${ }^{2}$ Molecular and \\ Cellular Biology Program, Fred Hutchinson Cancer Research Center and University of Washington, \\ Seattle, Washington 98109, USA
}

\begin{abstract}
Many laminated regions of the mammalian brain develop by the migration of neuronal precursor cells, whose final positions are coordinated by signals from the secreted molecule Reelin. Early events in Reelin signaling have been identified, but the mechanism of signal down-regulation has been unclear. A possible source of negative feedback is the Reelin-induced degradation of the critical intracellular signaling component, Disabled-1 (Dab1). Here we show that degradation of Dab1 depends on Dab1 phosphorylation at specific tyrosine residues and on the E3 ubiquitin ligase component Cullin 5 (Cul5). Cul5 forms complexes with SOCS (suppressors of cytokine signaling) proteins, which bind to phosphorylated Dab1 and target it for degradation in tissue culture cells. Ablation of Cul5 in migrating neurons causes an accumulation of active Dab1 protein and a unique cortical layering defect, characterized by excess migration and buildup of neurons at the top of the cortical plate. The results implicate Cul5 and SOCS proteins in down-regulation of Dab1 in vivo and show that Cul5 plays an essential role in regulating neuron migrations during cortical development, possibly by opposing a promigratory effect of Dab1.
\end{abstract}

[Keywords: Cullin; Dab1; Reelin; cortical development; neuronal migration; ubiquitin]

Supplemental material is available at http://www.genesdev.org.

Received August 13, 2007; revised version accepted September 14, 2007.

Development of complex organisms requires precise production, positioning, and differentiation of many cell types. In some cases, cells migrate long distances and along intricate routes to reach the places where they differentiate. This is the case during development of the mammalian neocortex (Hatten 2002; Marin and Rubenstein 2003; Nadarajah et al. 2003; Kriegstein and Noctor 2004). Neuronal progenitor cells, located in the ventricular zone adjacent to the lateral ventricle, give rise to precursors that migrate radially outward through a cell-poor region called the intermediate zone to establish the initial layers of the cortical plate. As embryonic development progresses, neuronal precursors will give rise to distinct types of projection neurons-distinguishable by their gene expression, morphology, axonal and dendritic architecture, and synaptic properties - that arrange themselves into six discrete layers that compose the mature cortical plate. These layers are normally arranged in an inside-out manner according to neuronal birth order. This arrangement is thought to have allowed for expansion of the neocortex during mammalian evolution, a process that would also require the evolution of mecha-

\footnotetext{
${ }^{3}$ These authors contributed equally to this work. ${ }^{4}$ Corresponding author.

E-MAIL jcooper@fhcrc.org; FAX (206) 667-6522.

Article is online at http://www.genesdev.org/cgi/doi/10.1101/gad.1604207.
}

nisms to direct the migration of each new layer of neurons past its predecessors (Rakic 1995; Kriegstein et al. 2006). As the cortex grows, neurons fated for the upper cortical plate layers must navigate through a thicket of other cells.

The neuron migrations that organize this inside-out layering of the neocortex are regulated by the ReelinDab1 pathway (for review, see Rice and Curran 2001; Gupta et al. 2002; Jossin 2004; Herz and Chen 2006). Reelin is a secreted protein that acts on other cells by inducing clustering of specific receptors, increasing the activity of two Src family tyrosine kinases (SFKs), Fyn and Src, and increasing tyrosine phosphorylation of the membrane-associated cytoplasmic protein Disabled-1 (Dab1). All these components have been shown by genetics and biochemistry to be involved in Reelin signaling in the neocortex, and failure of Reelin to induce Dab1 tyrosine phosphorylation during development results in an inversion of cortical plate layering.

While much is known about the initial events in Reelin signaling, less is known about how this signal is modulated. Negative feedback buffers signaling against noise and allows for adaptation, which is important for cells or axons to follow a concentration gradient during chemotaxis or to respond appropriately to temporal changes in stimulus strength (Ferrell 1996; Yi et al. 2000; Heinrich et al. 2002; Ming et al. 2002; Weiner 2002). 
While many signaling pathways are down-regulated by inactivation of a kinase, SFKs that have been activated by Reelin remain active due to positive feedback from phospho-Dab1 (Arnaud et al. 2003b; Ballif et al. 2003; Bock and Herz 2003). However, Reelin-induced tyrosine phosphorylation of Dab1 leads to polyubiquitination and proteasome-dependent degradation of Dabl (Arnaud et al. 2003a; Bock et al. 2004). Due to this degradation, Dab1 protein levels are lower in wild-type embryo brains than in Reelin, receptor, or SFK mutants (Rice et al. 1998; Trommsdorff et al. 1999; Howell et al. 2000; Kuo et al. 2005). After Reelin-induced down-regulation, Dab1 protein levels recover slowly, providing a refractory period during which the cells cannot respond a second time (Arnaud et al. 2003a; Bock et al. 2004). Reelin-induced degradation is a major mechanism for signal down-regulation, because proteasome inhibitors cause a sustained tyrosine phosphorylation of Dab1 in response to Reelin. These observations raise the possibility that degradation of tyrosine-phosphorylated Dabl is a negative feedback mechanism that could be important for regulating Reelin responses in vivo.

Here we investigated the mechanism for Dab1 degradation. Previous reports had shown that overexpression of the E3 ubiquitin ligase Cbl induces Dab1 ubiquitination in tissue culture cells (Suetsugu et al. 2004). However, degradation of the ubiquitinated Dab1 was not reported. In addition, Dab1 was found to bind and inhibit another E3 ligase, Siah-1A (Park et al. 2003). In contrast, we found that degradation of Dab1 in tissue culture cells is not induced by Cbl, but is specifically stimulated by overexpression of suppressor of cytokine signaling-1 (SOCS1) or SOCS3 proteins. We identified the tyrosine residues in Dab1 that mediate Reelin-induced Dab1 down-regulation in neurons and found that the same sites mediate SOCS-stimulated degradation in tissue culture cells. SOCS proteins are adaptors for an E3 ligase complex containing the key component Cullin-5 (Cul5) (for review, see Ilangumaran et al. 2004; Petroski and Deshaies 2005). Knockdown of Cul5 in cultured neurons protects active Dab1 from Reelin-induced degradation. Furthermore, Cul5 knockdown in developing mouse brain causes accumulation of phosphorylated, active Dab1 in migrating neurons, confirming the importance of Cul5 for Dab1 degradation in vivo. Cul5 knockdown also causes a novel upward shift in positions of cortical neurons, suggesting that ubiquitin-mediated proteolysis plays an important role in cortical lamination. This provides the first evidence that protein degradation is involved in coordinating neural migrations, and suggests that Reelin and Dabl stimulate migration, opposed by Cul5.

\section{Results}

Phosphorylation specificity of Reelin-induced Dab1 degradation

Previous studies implicated Reelin-induced SFK activation and Dab1 tyrosine phosphorylation in Dabl turnover (Arnaud et al. 2003a; Bock et al. 2004; Kuo et al.
2005). We were interested in which phosphotyrosines in Dab1 might be involved in turnover. Dab1 can be phosphorylated at up to four sites-a (Tyr185), b (Tyr198), c (Tyr220), and d (Tyr232)-by forced expression of an SFK (Fig. 1A; Howell et al. 2000; Keshvara et al. 2001). Phosphorylation of the b, c, and d sites has been detected in neurons (Keshvara et al. 2001; Ballif et al. 2004). The a and b sites lie in similar YQXI sequences, and the $\mathrm{c}$ and $\mathrm{d}$ sites lie in YXVP. To allow for possible redundancy between sites of similar sequence, we compared the Reelin-induced phosphorylation and degradation of wild-type Dab1 ${ }^{W T}, \mathrm{Dab}^{a b}$ (sites a and b mutated), Dab1 ${ }^{c d}$ (sites c and d mutated), and Dab1 ${ }^{5 F}$ (mutated at all four sites and Tyr200) (Howell et al. 2000). These proteins were tagged with GFP to distinguish them from endogenous Dab1, and expressed in primary neuron cultures using electroporation $(\mathrm{Xu}$ et al. 2005).

As shown in Figure 1B, phosphorylation of wild-type $\mathrm{Dab1}{ }^{W T}$-GFP is stimulated by Reelin, as expected. $\mathrm{Dab}^{a b}{ }_{\text {-GFP }}$ and Dab1 ${ }^{c d}$-GFP proteins exhibit reduced levels of basal and Reelin-stimulated tyrosine phosphorylation, but in both cases, Reelin still stimulates some phosphorylation of the remaining sites (Fig. 1B). This means that neither pair of phosphorylation sites is essential for phosphorylation of the remaining sites, at least when the proteins are overexpressed.

We then tested for Reelin-induced degradation, using MAP2-GFP as an internal control for electroporation efficiency. Cultures were exposed to Reelin for 3-5 d to induce Dab1 down-regulation. We found that Dab1 ${ }^{W T}$-GFP is down-regulated in Reelin-stimulated cultures relative to control cultures (Fig. 1C). By comparing the level of Dab1WT-GFP with MAP2-GFP in repeated experiments, we determined that the Reelininduced decrease was $\sim 30 \%$ (Fig. 1C, histogram). This compares with $\sim 80 \%$ decrease in endogenous Dab1 p80 in the same cultures (Fig. 1C, middle panel). The inefficient degradation of Dab1 ${ }^{W T}$-GFP relative to endogenous Dab1 p80 may be due to its overexpression and lower stoichiometry of phosphorylation (Xu et al. 2005). However, the degradation of Dab1 ${ }^{W T}$-GFP required phosphorylation, since levels of Dab1 $1^{5 F}$-GFP were equal in Reelin-treated and control cultures (Fig. 1C). Moreover, Dab1 ${ }^{a b}$-GFP was not degraded in response to Reelin, while Dab1 ${ }^{c d}$-GFP was degraded to the same extent at Dab1 ${ }^{W T}$-GFP. Testing the a and b phosphorylation sites individually showed that the $\mathrm{b}$ site is required for Reelin-induced degradation (Fig. 1C, histogram; Supplementary Fig. S1). These results show that a YQXI phosphorylation site is necessary and YXVP sites are dispensable for Reelin-induced degradation of Dab1, and suggest that the ubiquitination machinery recognizes pYQXI sites.

\section{SOCS proteins bind to and induce degradation} of phosphorylated Dab1

We had observed that Dab1 is constitutively tyrosinephosphorylated, but not degraded, when coexpressed 
A
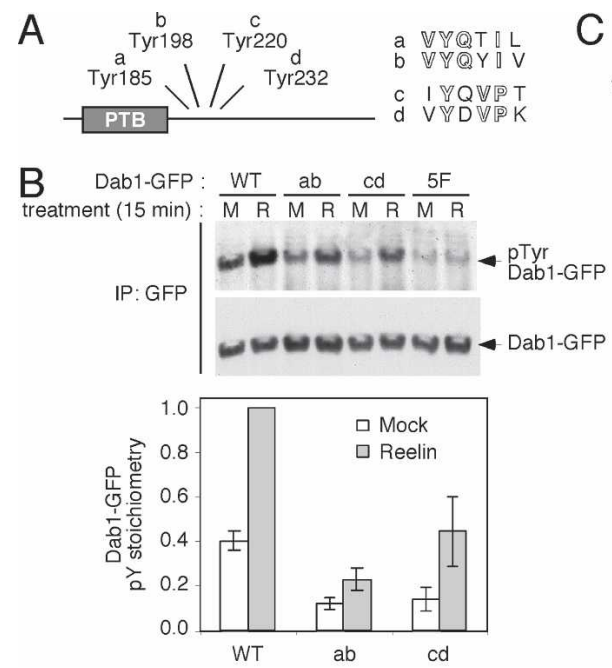
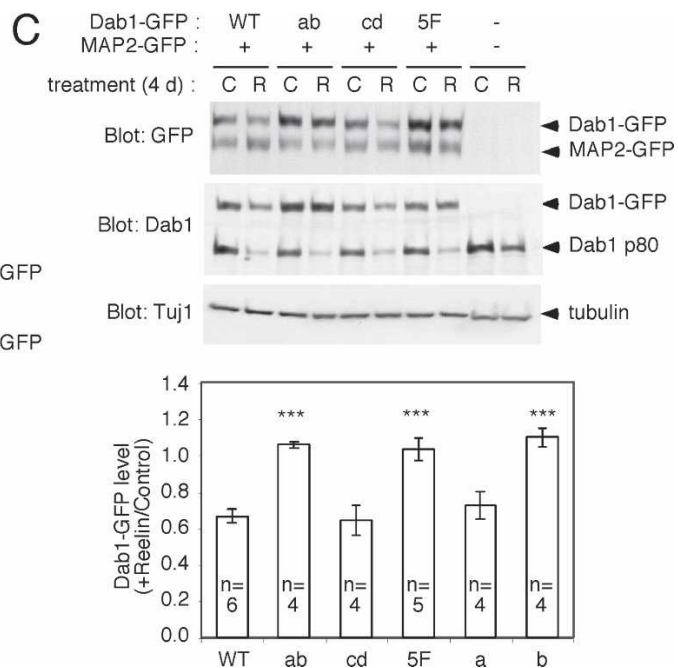

Figure 1. Specificity of Dab1 degradation in neurons. (A) Dab1 protein structure illustrating tyrosine phosphorylation sites studied. (B) Phosphorylation of wild-type and mutant Dab1 expressed in neuron cultures. Mouse cortical neurons were electroporated with either wild type or tyrosine-to-phenylalanine mutants of Dab1-GFP. On day 5 after electroporation, cells were treated for 15 min with mock or Reelin-containing supernatant and lysed. Cell lysates were immunoprecipitated with anti-GFP antibody and analyzed by Western blotting with anti-phosphotyrosine (4G10) or anti-Dab1 (B3) antibody. Dab1-GFP tyrosine phosphorylation relative to Dab1GFP protein was quantified by densitometry. Graph shows mean \pm SEM from four independent experiments. $(C)$ Effect of chronic exposure to Reelin on Dab1 levels. Neurons were coelectroporated with MAP2-GFP and wild-type or mutants of Dab1-GFP. Duplicate dishes were treated with Control (C; containing VLDLR ectodomain to sequester endogenous Reelin) or Reelin (R) for 4 d. Lysates were analyzed by Western blotting. The level of Dab1-GFP was normalized for transfection efficiency to MAP2-GFP, and the ratio in Reelin versus Control cultures was calculated. Graph shows mean \pm SEM from four to six independent experiments. $\left(^{\star \star \star}\right) P<0.005($ Student's $t$-test, two-tailed, relative to wild type).

with Fyn in transfected HEK293, HeLa, or COS cells (Howell et al. 2000; Arnaud et al. 2003b; data not shown). This suggests that a component of the machinery that degrades phosphorylated Dab1 is absent or limiting in some nonneuronal cell lines. Therefore, we used COS cells as a system for testing the activity of candidate E3 ligases against phosphorylated Dab1.

We obtained expression vectors for various E3 ligases, including Cbl, Cbl-b, Cbl-3, Hakai, E6AP, SOCS1, SOCS2, SOCS3, LNX, and Rnf5 (see the Supplemental Material). These were cotransfected with Dabl-GFP and Fyn into COS cells. GFP was used to normalize transfection efficiency. Protein levels were assessed $24 \mathrm{~h}$ later by Western blotting. While most E3 ligases tested did not cause a significant decrease in Dab1-GFP relative to GFP, expression of SOCS1 caused a dose-dependent decrease in Dab1-GFP (Fig. 2A; Supplementary Fig. S2). By use of $\left.{ }^{35} \mathrm{~S}\right]$ methionine pulse-chase followed by immunoprecipitation, we found that SOCS1 stimulated Dab1-GFP degradation approximately threefold (Fig. 2B). Degradation was also induced by SOCS2 and SOCS3 (Fig. 2C). SOCS1 targets GFP-tagged or untagged Dab1 and does not target Dab2 under the same conditions (Fig. 2D). Moreover, SOCS proteins can bind to Dab1, as shown by coimmunoprecipitation of Dab1-GFP with Flag-tagged SOCS1 or SOCS3 from cotransfected HeLa cells (Fig. 2E). These results indicate that overexpressed SOCS proteins can bind to Dab1 and target it for degradation in nonneuronal cell types.

\section{Phosphorylation specificity of SOCS-mediated Dab1 degradation}

SOCS1-3 contain SH2 domains through which they bind specific phosphotyrosine sites in their substrates (Krebs and Hilton 2000; De Souza et al. 2002; Hirano and Murakami 2006). Reelin-induced Dab1 degradation in neurons requires the YQXI phosphorylation site (Fig. 1C). Therefore we tested whether SOCS-mediated degradation of Dab1 in transfected cells also requires phosphorylation, and which sites are involved. Indeed, Dab1 $1^{W T}$. GFP was degraded more with wild-type Fyn ${ }^{W T}$ than with kinase inactive Fyn ${ }^{K M}$ (Fig. 3A). Dab1 ${ }^{W T}$-GFP was degraded more than nonphosphorylated Dab1 ${ }^{5 F}$-GFP (Fig. 3B). Moreover, Dab1 ${ }^{\text {cd }}$-GFP was degraded more rapidly than Dab1 ${ }^{a b}$-GFP (Fig. 3B), mimicking their degradation in neurons. We also noted that Fyn levels were reduced approximately in parallel with Dabl, suggesting that SOCS1 may target a complex of active Fyn and Dab1 phosphorylated at the ab sites (Fig. 3A,B). However, in neurons, SFK levels are not significantly reduced by Reelin stimulation (Arnaud et al. 2003a). This suggests either that neurons contain more SFK molecules than Dab1 molecules, so a decrease in SFK level is too small to detect, or that SFKs are not codegraded with Dab1 in Reelin-stimulated neurons.

Consistent with the degradation results, binding of Flag-SOCS1 to Dab1-GFP required Fyn activity and the ab phosphorylation sites in Dab1, with the b site essen- 


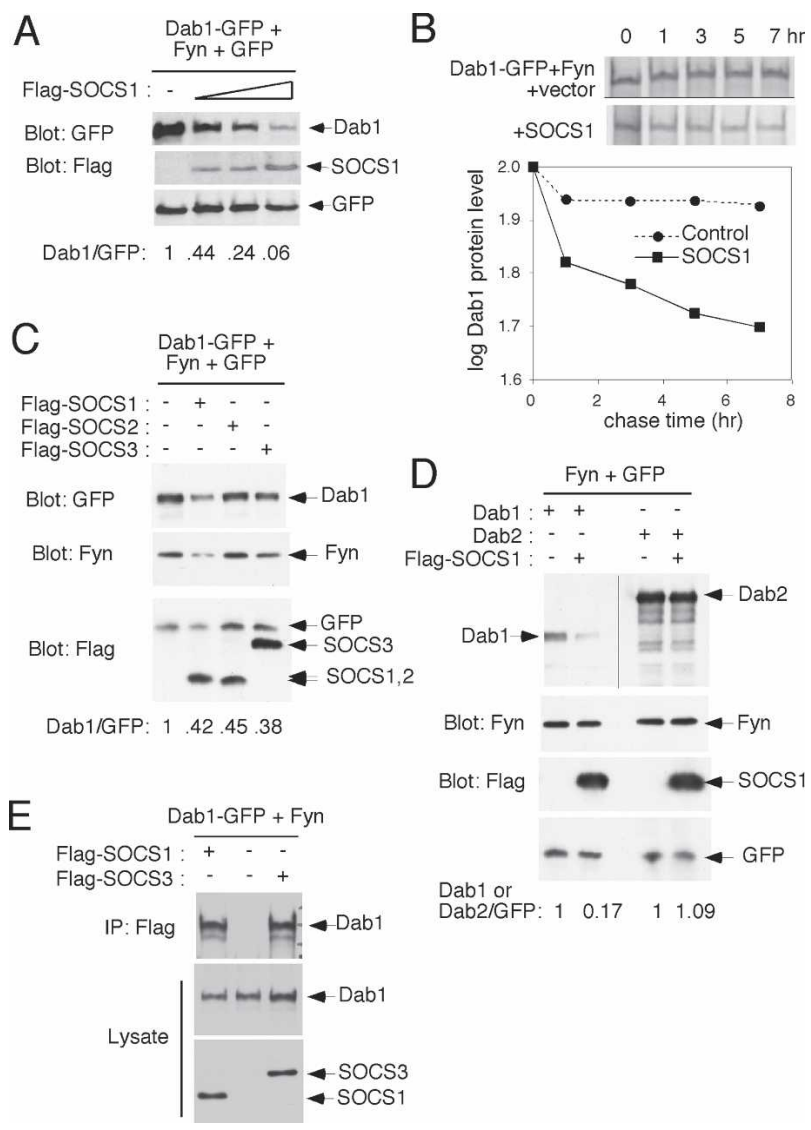

Figure 2. SOCS proteins mediate degradation of Dab1 in nonneuronal cells. (A) Increasing SOCS1 progressively decreases Dab1. COS cells transfected with Dab1-GFP, Fyn ${ }^{W T}$, Flag-GFP, and increasing amounts of Flag-SOCS $1(0.25 \mu \mathrm{g}, 0.5 \mu \mathrm{g}, 1 \mu \mathrm{g})$ were lysed and analyzed by Western blot using anti-GFP and anti-Flag antibodies. The ratio of Dab1-GFP to GFP is shown. $(B)$ SOCS1 decreases Dab1 half-life. COS cells transfected with Dab1-GFP together with Fyn ${ }^{W T}$ and Flag-SOCS1 or control vector were pulse-labeled with $\left[{ }^{35} \mathrm{~S}\right]$-Met and $\left[{ }^{35} \mathrm{~S}\right]$-Cys for $45 \mathrm{~min}$, and then chased for the indicated times (see Supplemental Material). Dab1-GFP was immunoprecipitated with anti-GFP, and radioactivity was quantified. (C) SOCS1 and SOCS3 reduce Dab1 levels. COS cells were transfected with Dab1-GFP together with Fyn ${ }^{W T}$, Flag-GFP, and Flag-SOCS1, Flag-SOCS2, or Flag-SOCS3 and analyzed by Western blot. $(D)$ Degradation of Dab1 is specific and does not require the GFP tag. COS cells transfected with Fyn ${ }^{W T}$, Flag-GFP, and Dab1 (lanes 1,2) or Dab2 (lanes 3,4) in the presence of Flag-SOCS1 or control vector were lysed and analyzed as above, except using anti-Dabl and antiDab2 antibodies. (E) SOCS1 and SOCS3 bind to Dab1. HeLa cells were transfected with Dab1-GFP, Fyn ${ }^{W T}$, and Flag-SOCS1 or Flag-SOCS3 or vector. Lysates were immunoprecipitated with anti-Flag, and bound Dabl-GFP was detected using antiGFP antibody. Equal expression was confirmed by Western blot.

tial (Fig. 3C,D). These binding and degradation assays show that SOCS1 targets Dabl after it is phosphorylated by Fyn at the YQXI sites. This suggests that SOCS proteins and the E3 ubiquitin ligase operative in neurons have the same specificity.
Role of Cul5 in Reelin-induced degradation of Dab1 in neurons

At least five SOCS proteins are expressed in developing brain, including SOCS1-3, SOCS7, and CIS (Polizzotto et al. 2000; Krebs et al. 2004). Therefore, we suspected that SOCS proteins might be redundant for Reelin-induced Dab1 degradation, and the effects of inhibiting any one SOCS protein may be undetectable. SOCS proteins are members of multiprotein E3 ligase complexes (Fig. 4A; Krebs and Hilton 2000; Ilangumaran et al. 2004; Petroski and Deshaies 2005). They contain a "SOCS-box" sequence that binds to ElonginBC and a Cullin, Cul2 or Cul5. The Cullin, in turn, binds to RING-finger proteins $\mathrm{Rbx} 1$ or Rbx2 and forms the catalytic core for ubiquitin transfer. Several researchers have addressed whether Cul2 or Cul5, or both, complex with SOCS proteins. Kamizono et al. (2001) found that overexpression of a mutant Cul2 inhibits SOCS1-stimulated degradation of a tyrosine-phosphorylated substrate. However, Cul2 and Cul5 are closely related, and the overexpressed Cul2 mutant may displace either Cul2 or Cul5. On the other hand, Kamura et al. (2004) showed that Cul5 coimmunoprecipitates with various SOCS proteins from HeLa cells, while Cul2 coimmunoprecipitates with a different substrate recognition protein, VHL, which lacks a SOCSbox.

We used in situ hybridization to assess expression of Cul2 and Cul5 in embryonic brain. Both genes are expressed in the ventricular zone and cortical plate (Supplementary Fig. S3). To test whether either Cul2 or Cul5 might be involved in Dab1 degradation in response to Reelin, we used Cul2 and Cul5 short hairpin RNA (shRNA) constructs (Kamura et al. 2004). Embryonic cortical neurons were coelectroporated with Dabl-GFP, internal control MAP2-GFP, and various shRNA constructs including Cul2, Cul5, or empty vector (EV). Cultures were split and exposed to control medium or Reelin to induce Dab1 down-regulation (as in Fig. 1C). Relative levels of Dab1-GFP were measured by Western blotting in replicate experiments (Fig. 4B). While Reelin caused a $50 \%-60 \%$ reduction in Dab1-GFP levels if empty vector or Cul2 shRNA was used, coelectroporation of Cul5 shRNA significantly protected Dab1-GFP from Reelin-induced degradation. Western blotting for Cul5 and Cul2 confirmed that both proteins are expressed in cortical neurons and their expression is inhibited by the respective shRNAs (Fig. 4C). We also noted a partial protection of endogenous Dab1 by either Cul5 shRNA or by an shRNA directed against a different sequence in Cul5 (Cul5' shRNA) (Fig. 4C). Incomplete protection was expected, since the electroporation efficiency is $40 \%-70 \%$. As a control for possible off-target effects of the Cul5 shRNA, we coelectroporated a Cul5* plasmid that encodes wild-type Cul5 protein but is resistant to the Cul5 shRNA (Supplementary Fig. S4). Cul5 shRNA no longer protected endogenous Dab1 from degradation if $\mathrm{Cul5}{ }^{\star}$ protein was coexpressed (Fig. 4D). Control experiments showed that Cul5 shRNA did not affect Dab1 mRNA levels in cultured neurons (Supplementary 


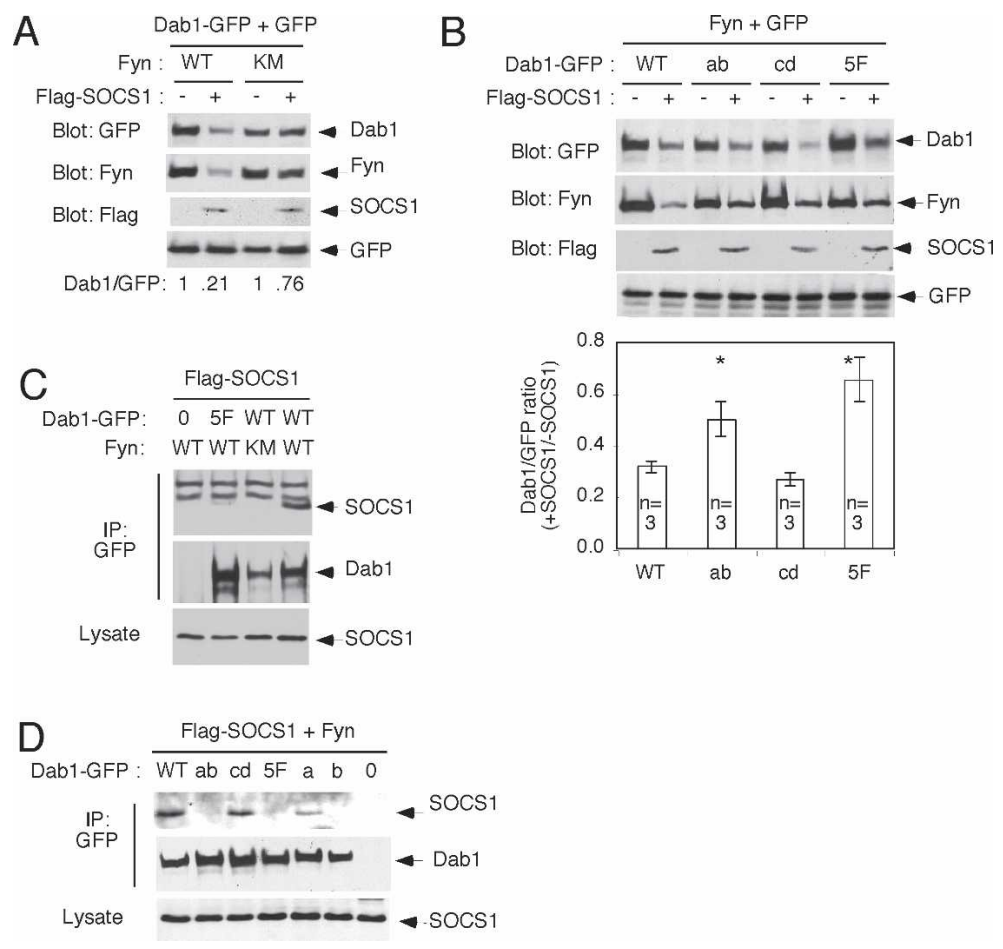

Figure 3. Specific Dabl tyrosine phosphorylation sites target SOCS-mediated degradation. (A) Degradation of Dab1 in COS cells requires kinase-active Fyn. COS cells were transfected with Dab1-GFP, Fyn ${ }^{W T}$, Fyn $^{K M}$ (kinase dead), GFP, and Flag-SOCS1 or vector. Lysates were analyzed by Western blot. (B) Mutation of $\mathrm{a}$ and $\mathrm{b}$ phosphorylation sites in Dabl inhibits SOCS-induced degradation. COS cells were transfected with Fyn ${ }^{W T}$, GFP, wild-type, or mutant Dab1GFP and Flag-SOCS1 or control vector. Lysates were analyzed by immunoblot. Dabl-GFP levels were normalized to GFP for transfection efficiency, and the ratio in the presence versus absence of SOCS1 was calculated. Graph shows mean \pm SEM from three independent experiments. $\left(^{\star}\right) P<0.05$. $(C)$ Binding of Dab1 to SOCS1 requires phosphorylation. HeLa cells were transfected with wild-type or mutant Dab1-GFP, wild-type or mutant Fyn, and SOCS1. Lysates were immunoprecipitated with anti-GFP and analyzed with anti-Flag antibodies. (D) Importance of different phosphorylation sites for binding. Transfected HeLa cells were analyzed as above, except anti-SOCS1 antibodies were used.
Fig. S5A). These results show that Cul5 is important for Reelin-induced post-transcriptional down-regulation of Dab1 in neuron cultures.

To gain further evidence whether Cul5 is involved in Dab1 down-regulation, we tested whether Dab1 and Cul5 interact in neurons. Indeed, a small amount of Cul5 was detected in immunoprecipitates of Dab1 from Reelin-stimulated but not control neurons (Fig. 4E).

Taken together, our results suggest that Reelin induces Dab1 tyrosine phosphorylation at the YQXI sites, which recruits one or more SOCS proteins, which in turn initiates Dabl ubiquitination and degradation via Cul5.

\section{Cul5 knockdown protects Dab1 from degradation in vivo}

To test the role of Cul5 in Dab1 down-regulation in vivo, and to assess the possible role of Cul5 in neuron positioning, we used in utero microinjection and electroporation (Tabata and Nakajima 2001). Cul5 shRNA or corresponding vector was mixed with a GFP expression plasmid in a 4:1 ratio and microinjected into the lateral ventricles of embryonic day 14.5 (E14.5) mouse embryos, followed by electroporation to induce DNA uptake into neuronal progenitors in the ventricular zone. The embryos were allowed to develop in utero until E17.5 or E19.5, then brains were recovered by dissection, and the progeny of electroporated progenitors were identified using GFP antibodies.

Immunofluorescence with anti-Dab1 antibodies revealed a marked increase in Dabl levels in GFP-expressing cells in embryos that received Cul5 shRNA (Fig. 5A) compared with controls receiving vector alone (Fig. 5B). The increase was specific because there was no increase in Dab1 in neurons that did not express GFP, or in neurons expressing Cul2 shRNA (Fig. 5D). Increased Dab1 was also detected with an independent Dabl antibody (Fig. 5C). In situ hybridization showed that Dab1 mRNA levels were not increased by Cul5 shRNA (Supplementary Fig. S5B). The increase in Dab1 immunofluorescence was similar to that observed previously in Reelin and Src/Fyn mutant mice, where Dab1 degradation is inhibited because upstream signaling is blocked (Rice et al. 1998; Kuo et al. 2005), but here the undegraded Dab1 is presumably active. Indeed, antibodies to phosphorylated Dab1 (Dab1pY220) (Keshvara et al. 2001) detected increased active Dab1 in neurons receiving Cul5 shRNA but not controls (Fig. 5E,F). Therefore, Cul5 inhibition increases levels of phosphorylated Dab1 in vivo.

\section{Cul5 knockdown alters neuron positioning}

We noted that Cul5 shRNA-expressing neurons were conspicuously shifted to a more superficial position relative to controls, when studied at E19.5, $5 \mathrm{~d}$ after injection (Figs. 5, 6A). While control neurons had reached the outer edge of the cortical plate and then been displaced to a deeper position in layer III/IV by younger neurons destined for layers II/III, Cul5 shRNA neurons remained high in the cortical plate. They were still superficial $7 \mathrm{~d}$ after electroporation, at postnatal day 2 (P2) (Supplementary Fig. S6). At E19.5, the affected neurons are at the very top of the cortical plate, and some are within the calretinin-positive processes of Cajal-Retzius cells in the marginal zone, but we did not detect movement beyond the somata of the Cajal-Retzius neurons (Fig. 7A). 
Figure 4. Role of Cul5 in Reelin-induced Dab1 degradation in neurons. (A) Components of the SOCS-associated E3 ligase complex. (B) Cul5 shRNA inhibits Reelin-induced degradation of Dab1-GFP in neuron cultures. Cortical neurons were electroporated with Dab1-GFP, MAP2-GFP, and $6 \mu \mathrm{g}$ of indicated shRNA constructs, except for $\mathrm{Cul5}^{\#}$, where $4 \mu \mathrm{g}$ were used. Duplicate dishes were treated with Control (C) or Reelin (R) for $4 \mathrm{~d}$. Lysates were analyzed using anti-GFP (top), anti-Dab1, anti-Cul5, and anti-Tuj1 (bottom). Dab1-GFP levels in Reelin versus control cultures were calculated as in Figure 1B. Graph shows mean \pm SEM from three independent experiments. $\left({ }^{\star \star}\right) P<0.01 .(C)$ Cul5 shRNA inhibits degradation of endogenous Dab1. Cortical neurons were electroporated with shRNA (6 $\mu \mathrm{g})$ against Cul2 or two sequences in Cul5 and treated with Control (C) or Reelin (R) for $4 \mathrm{~d}$. Graph shows mean \pm SEM effect of Reelin on Dab1 levels (normalized to Tuj1) from two to three independent experiments. $\left({ }^{*}\right) P<0.05$. (D) Control for off-target effects of Cul5 shRNA. Cortical neurons were electroporated with Cul5 shRNA and rescue construct Cul5*, and analyzed as above. The myc-tagged Cul5* was resolved from endogenous Cul5. $(E)$ Cul5 binds to Dab1 in Reelinstimulated neurons. Cortical neurons were treated for 15 min with mock or Reelin-containing supernatant. Dab1 protein was immunoprecipitated by anti-Dab1 (E19) and analyzed using anti-Cul5 and anti-Dab1 (B3).
A

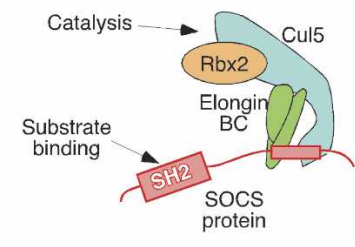

C shRnA: EV Cul2 Cul5 $\frac{\text { Cul5' }}{C}$

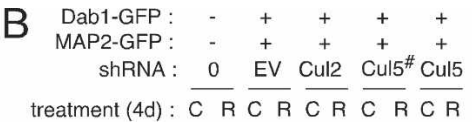

Blot: GFP $\quad=-\equiv=E=- \pm$ Dab1-GFP

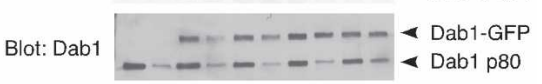

Blot: Cul5 - - - - - - - 4 Cul5

Blot: Tuj1 _- tubulin
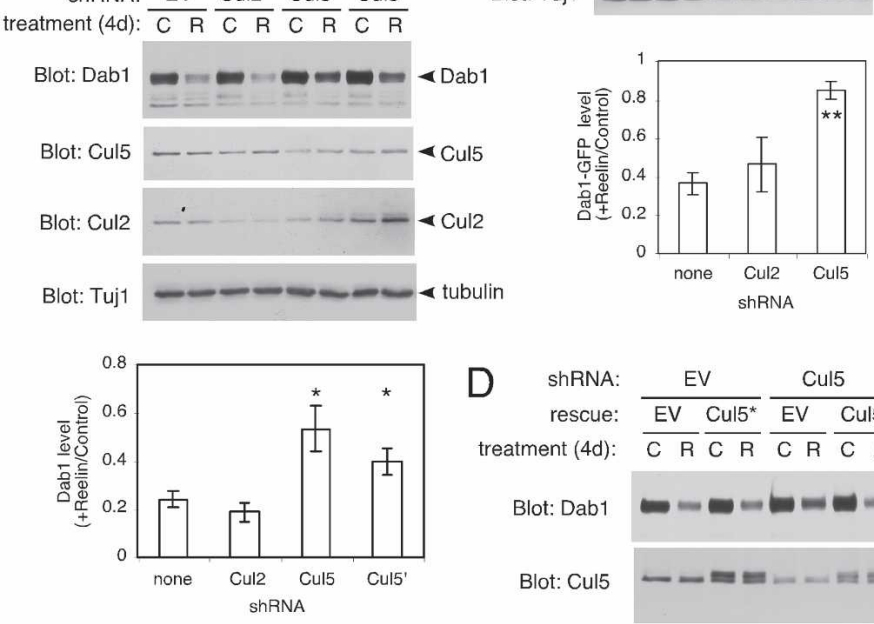

$E$

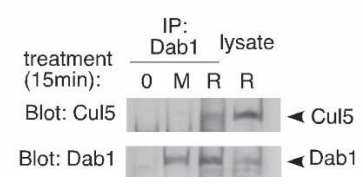

We quantified neuron positions by dividing the outer part of the cortex into eight divisions, $\sim 50 \mu \mathrm{m}$ thick (Fig. 6A). Division 1 is the marginal zone; divisions 2-7, the cortical plate; and division 8 , the top of the intermediate zone. GFP-expressing neurons in each division were counted for several sections from each embryo, and the percentage of cells in each division was calculated. Data from replicate embryos were averaged, and the mean and standard error were plotted (Fig. 6B). For each DNA, we calculated the mean neuron position and the significance of any change, as described in Materials and Methods. Mean neuron positions for this and subsequent experiments are provided in the figure legends and summarized below in Figure 8D. We found that the effects of Cul5 and Cul5' shRNAs on neuron positioning were highly significant when compared with vector $(P=0.002$ and 0.018 , respectively). As a further control, Cul2 shRNA had no significant effect on position (Fig. 8D; Supplementary Fig. S7A).

To confirm the specificity of Cul5 knockdown, we restored Cul5 expression using the shRNA-resistant Cul5* mRNA. This construct or the corresponding vector was mixed with Cul5 shRNA plasmid or matched vector in a 1:1 ratio and then injected and analyzed at E19.5. Reexpression of Cul5* together with Cul5 shRNA rescued neuron positioning $(P=0.007)$ (Figs. 6C, 8D). This confirms that displacement of Cul5 shRNA-expressing neu- rons is due to knockdown of Cul5 and not off-target effects.

A superficial position could result if the first neuronal progeny derived from Cul5 shRNA-expressing progenitors were selectively lost or GFP-negative, so that only later-born progeny were detected. However, when BrdU was used to label dividing cells at the time of electroporation with Cul5 shRNA, double BrdU- and GFP-labeled neurons were found at the top of the cortical plate at E19.5 (Supplementary Fig. S7B). Moreover, the GFP-labeled Cul5 shRNA neurons lay above neurons of the same birth date labeled only with BrdU (Supplementary Fig. S7C). We think it is unlikely that possible effects of Cul5 shRNA on cell proliferation alter our results, since essentially all GFP marker has been diluted out of the progenitor cells by $2 \mathrm{~d}$ after electroporation (data not shown). The GFP plasmid, and presumably shRNA, are only retained in the post-mitotic, migratory cells. In addition, Cul5 shRNA-expressing cells detected at E19.5 were neurons because they expressed Tuj1 and their axons could be detected coursing through the intermediate zone (Fig. 7B,C). Moreover, the cells expressed the upper layer marker Brn1 and not the lower layer marker Tbr1, as expected for neurons born after E14.5 (Fig. 7D,E). Thus, Cul5 shRNA affects Dab1 protein level but appears not to affect cell fate. 

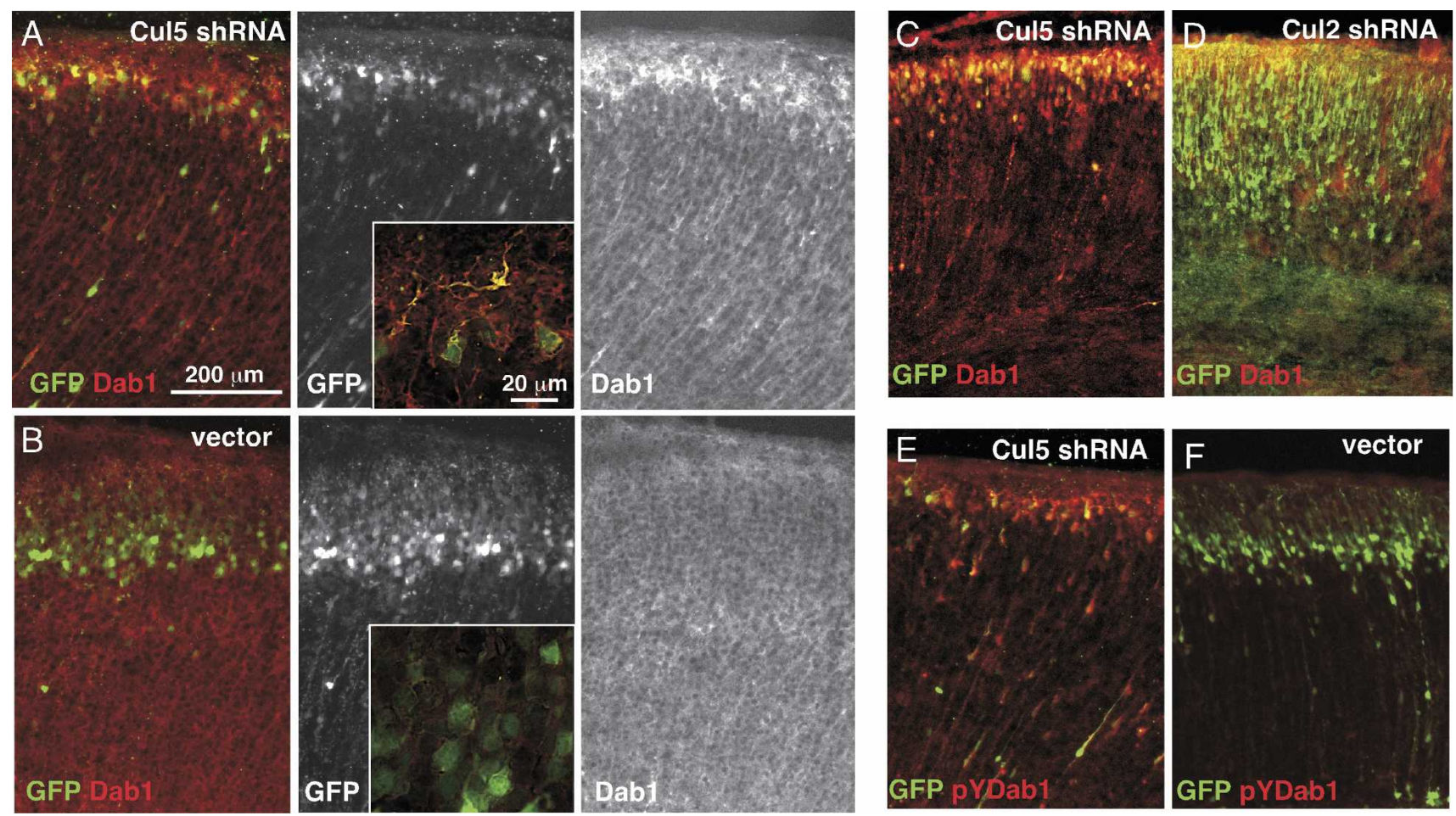

Figure 5. Effects of Cul5 shRNA on Dab1 protein level in vivo. Embryos were microinjected and electroporated with $2 \mu \mathrm{g}$ of Cul5 shRNA $(A, C, E)$, vector $(B, F)$, or Cul2 shRNA $(D)$ and $0.5 \mu \mathrm{g}$ of GFP plasmid pCAGIG at E14.5. Sections were prepared at E19.5 and stained with mouse anti-GFP (green) and rabbit anti-Dab1 (red) $(A, B)$, rabbit anti-GFP and goat anti-Dab1 (red) $(C, D)$, or mouse anti-GFP and rabbit anti-pY220 Dab1 (red) $(E, F)$. Slides were stained and imaged in parallel with a 10x objective. Inserts show Deltavision images using a $40 \times$ oil objective.

The superficial location at E19.5 and P2 could be due to changes in migration rate. Cul5 shRNA may retard migration, such that E14.5 neurons only just arrive at the top of the cortical plate at E19.5. Alternatively, Cul5 shRNA may not reduce the velocity of migration, but may prevent the cells that reach the marginal zone from stopping and letting their younger siblings go by. To distinguish these possibilities, embryos microinjected at E14.5 were analyzed $3 \mathrm{~d}$ later. At this time, both Cul5 shRNA and control neurons were found in the outer part of the cortical plate, with Cul5 shRNA neurons more superficial than control, indicating that Cul5 shRNA increases neuronal migration (Fig. 6D). The continued presence of Cul5 shRNA neurons at the top of the cortical plate at E19.5 or P2 implies that they do not let their younger siblings past.

\section{Dab1 role in Cul5-regulated neuron positioning}

Previous investigations have shown that neurons migrate more slowly in Dab1 mutant brains and that Dab1 mutant neurons layer below wild-type neurons in mosaic embryos (Hammond et al. 2001; Tabata and Nakajima 2002). In addition, in utero electroporation of Dab1 shRNA at E18 caused neurons to lag behind their siblings, and their leading processes were less developed (Olson et al. 2006). In utero infection of wild-type or
Dab1 mutant brains with retroviruses encoding phosphorylation site mutants of Dab1 allowed the role of Dab1 phosphorylation in the movement of individual neurons to be tested (Sanada et al. 2004). Dab1 mutants that were unable to rescue movement were found deeper in the cortical plate and were closer to their radial glia guides than control neurons. These results suggest that Dab1 regulates the speed of migration, final position, and radial glia adhesion.

We first checked the effect of Dab1 shRNA on neuron position. Embryos were electroporated with a mixture of Dab1 shRNAs at E14.5. Five days later, at E19.5, Dab1 shRNA neurons were significantly deeper than matched vector controls $(P=0.011)$ (Fig. 8A, B,D), as expected (Hammond et al. 2001; Tabata and Nakajima 2002; Sanada et al. 2004; Olson et al. 2006). Dab1 shRNA neurons were also slightly deeper than controls at E17.5 (data not shown). We then tested whether Dab1 knockdown would interfere with the overmigration of Cul5 shRNA neurons. We injected Cul5 shRNA, GFP tracer, and either vector or Dab1 shRNA at E14.5 and scored neuron positions at E19.5. Dab1 shRNA ameliorated the superficial shift caused by Cul5 shRNA $(P=0.0025)$ (Fig. $8 \mathrm{C}, \mathrm{D})$. However, we did not detect true epistasis. Double knockdown neurons were in a similar position to normal neurons (Fig. 8D). If Cul5 acted solely to inhibit Dab1 destruction, and if high Dab1 levels were the sole cause of ectopic neuron positioning, then double knockdown 
Feng et al.
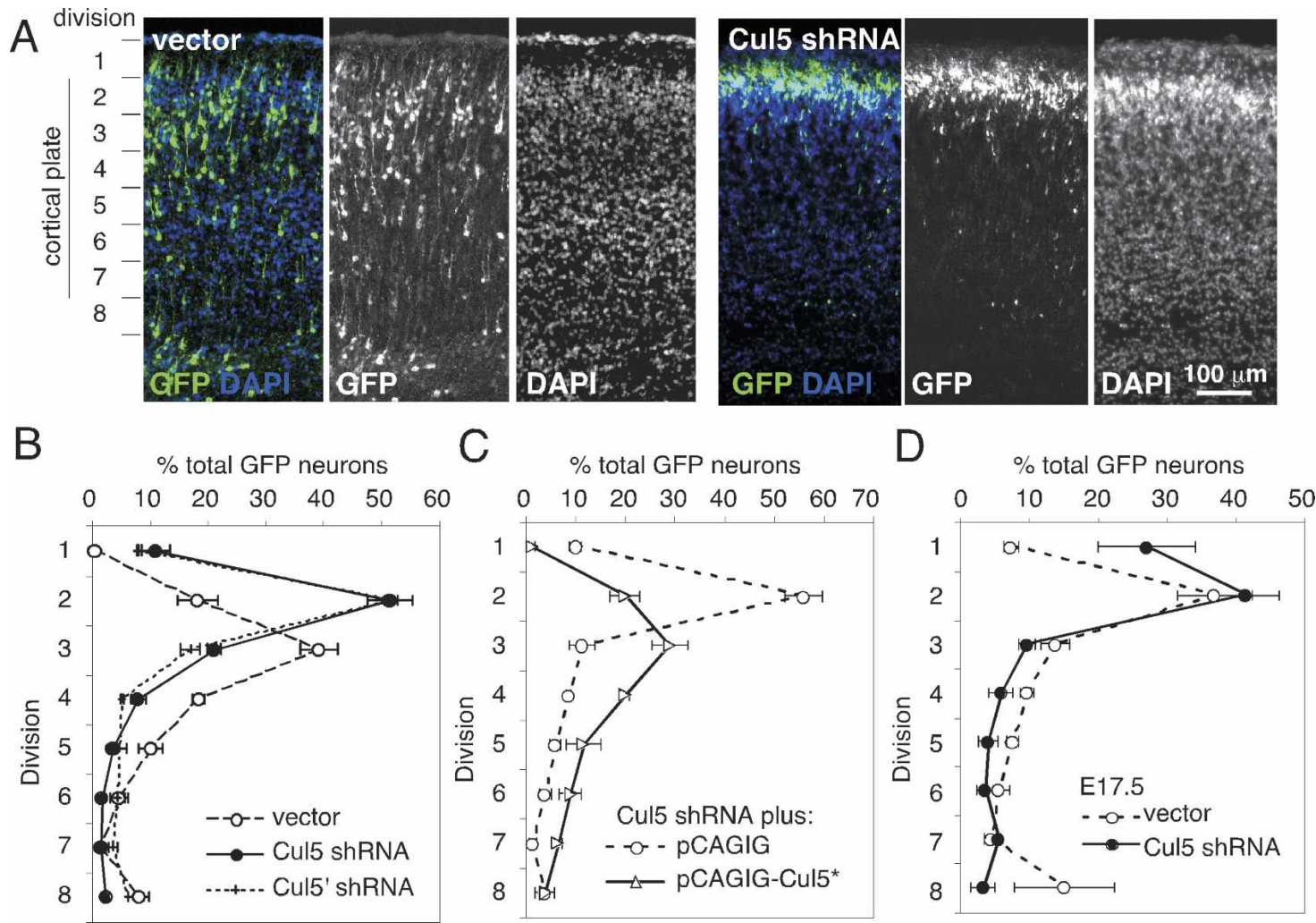

Figure 6. Effects of Cul5 shRNA on neuron positioning. (A) Positions of GFP-positive neurons at E19.5, after in utero microinjection at E14.5 with $2 \mu \mathrm{g}$ of vector or Cul5 shRNA and $0.5 \mu \mathrm{g}$ of GFP plasmid pCAGIG. Cortical divisions used for quantification are indicated on the left. (B) Quantification of neuron positions at E19.5. Mean \pm SEM for $n=5$ vector, $n=5$ Cul 5 shRNA, and $n=2$ Cul5' shRNA embryos. Average neuron positions were vector, $3.79 \pm 0.19$; Cul5 shRNA, $2.64 \pm 0.08(P=0.002)$; and Cul5' shRNA, $3.04 \pm 0.10$ $(P=0.018) .(C)$ Rescue of Cul5 knockdown by coexpressing Cul5 ${ }^{\star}$. Embryos were injected with $1 \mu \mathrm{g}$ of Cul5 shRNA and $1 \mu \mathrm{g}$ of either pCAGIG or pCAGIG-Cul5*, and scored on E19.5. Mean \pm SEM for $n=3$ vector and $n=4$ Cul5* embryos. Average neuron positions were Cul5 shRNA plus pCAGIG vector, $2.79 \pm 0.18$; and Cul5 shRNA plus pCAGIG-Cul5*, $3.91 \pm 0.17(P=0.007)$. $(D)$ Cul5 shRNA has little effect on migration rate. Positions at E17.5. Mean \pm SEM for $n=4$ vector and $n=3$ Cul5 shRNA embryos. Average neuron positions were vector, $3.83 \pm 0.43$; and Cul5 shRNA, $2.68 \pm 0.0 .23(P=0.087)$.

of Cul5 and Dab1 should cause the same phenotype as Dab1 shRNA; i.e., deeper position. Therefore, the results suggest that Dabl contributes to the effect of Cul5 knockdown but the pathway is probably not linear.

\section{Discussion}

We report the following major findings. First, our results suggest that SOCS proteins and Cul5 mediate negative feedback in Reelin signaling by targeting phosphorylated Dab1 for polyubiquitination and degradation. When Dab1 degradation is inhibited in vivo by Cul5 knockdown, tyrosine-phosphorylated Dab1 protein accumulates. Second, we found a novel function for Cul5, inhibiting overmigration of cortical neurons. When Cul5 is inhibited, neurons migrate at normal or slightly increased speed to the top of the cortical plate, and remain at the top as younger neurons accumulate below them. This phenotype is partly mediated by the increased Dab1 levels, since it is neutralized by inhibiting Dabl expression. This finding reveals Cul5 as an important regulator of neuron positioning during cortical development, possibly by opposing Dab1.

\section{Mechanism of Dab1 degradation}

Our results suggest a model for down-regulation of Dab1 in which Reelin induces Dab1 phosphorylation at several sites by Src family kinases, the phosphorylated pYQXI site Tyr198 is then recognized by one or more SOCS proteins, and an elonginB/C-Cul5 complex is recruited to polyubiquitinate Dabl and direct it to the proteasome (Fig. 8E). Three lines of evidence support this model. First, we showed that SOCS1, SOCS2, or SOCS3 could target Dab1 for degradation in tissue culture cells dependent on Fyn. Cbl family proteins, previously implicated in Dab1 ubiquitination based on overexpression experiments (Suetsugu et al. 2004), seem not to be involved. Second, the tyrosine phosphorylation sites required for SOCS-mediated degradation and for binding to SOCS proteins are the pYQXI sites, primarily if not exclusively Tyr198, that are also required for Reelin-stimulated degradation of Dab1 expressed in neurons. This sequence specificity is compatible with the known binding specificity of isolated SOCS SH2 domains (De Souza et al. 2002; Hirano and Murakami 2006). Third, Cul5 shRNA inhibited Reelin-induced Dab1 degradation in 

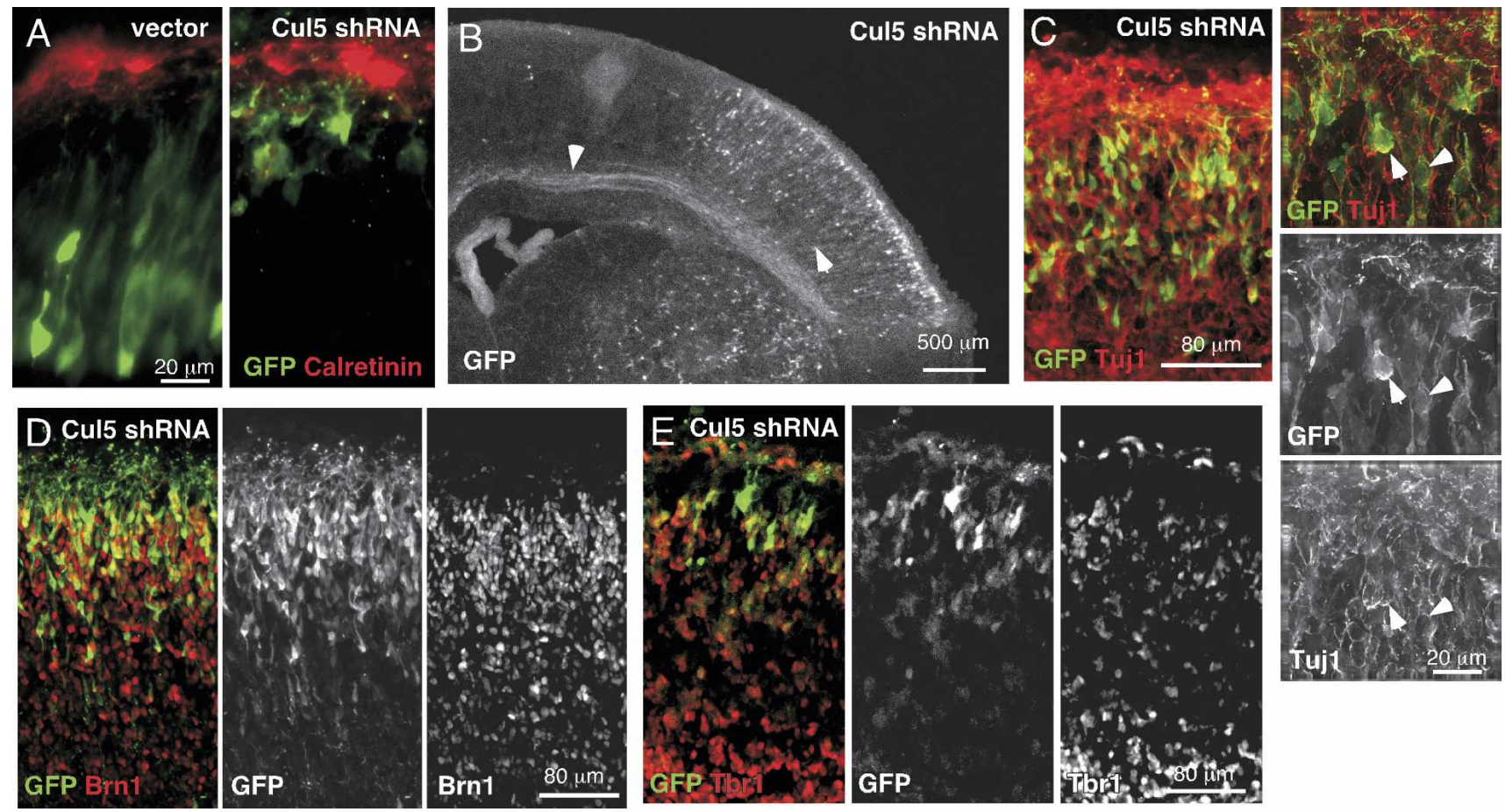

Figure 7. Characterization of Cul5 shRNA-expressing neurons. (A) Cul5 shRNA neurons lie at the bottom of the marginal zone. GFP-positive neurons at E19.5, after in utero microinjection at E14.5 with $2 \mu \mathrm{g}$ of vector or Cul5 shRNA and $0.5 \mu \mathrm{g}$ of GFP plasmid pCAGIG. Stained with antibody to calretinin (red). (B) Cul5 shRNA neurons produce axons. Low-power view, showing cortico-calosal axons coursing through the intermediate zone (arrowheads). (C) Cul5 shRNA neurons express neuron-specific $\beta$-tubulin. (Green) Rabbit anti-GFP; (red) mouse Tuj1. High-power images were taken with a 40x oil immersion lens and subjected to deconvolution (0.5- $\mu \mathrm{m}$ z-series, stack of 35 images). (D) Staining for upper-layer marker, Brn1. Sections stained at E17.5. (Green) Rabbit anti-GFP; (red) hamster anti-Brn1. (E) Staining for lower-layer marker, Tbr1. Sections stained at E17.5. (Green) Chicken anti-GFP; (red) rabbit antiTbr1.

cultured neurons and raised Dab1 protein levels in neurons in the developing brain. However, we did not identify which SOCS protein targets Dab1 in neurons. The large number of SOCS genes expressed during brain development means that they may have overlapping functions (Polizzotto et al. 2000; Krebs et al. 2004). Indeed, brain phenotypes have not been reported in SOCS1 and SOCS3 knockouts, and knockout of SOCS2 in the brain inhibits neurogenesis but not cortical layering (Turnley et al. 2002). We therefore suspect that there may be redundancy between SOCS proteins for Dab1 degradation. Future studies using compound mutants or combinations of shRNAs may be required to identify which SOCS proteins are involved in Dabl degradation in vivo.

It is surprising that SOCS-Cul5 complexes are involved in Reelin-activated SFK signaling during development, because SOCS proteins have been most extensively studied as cytokine-induced genes that inhibit JAK family tyrosine kinases and their substrates (Krebs and Hilton 2000; Ilangumaran et al. 2004). However, SOCS genes are quite widely expressed, including in developing and adult brain (Polizzotto et al. 2000; Krebs et al. 2004). Also, SOCS1 was previously found to bind to Fyn and to target a SFK phosphorylation site in focal adhesion kinase (De Sepulveda et al. 1999; Liu et al. 2003). Our finding that SOCS1 targets both Dab1 and overexpressed Fyn for degradation suggests that SOCSCul5 complexes may down-regulate other SFK signaling events in neurons. It is also possible that SOCS-Cul5 activity is regulated by post-translational modification. Cul5 is regulated by neddylation, and SOCS proteins are themselves subject to tyrosine phosphorylation (Petroski and Deshaies 2005). Thus the SOCS-Cul5 complex may be a nexus for integrating signaling events that lead to down-regulation of tyrosine kinase pathways.

Inhibiting Cul5 function causes a novel overmigration of neurons

In utero electroporation has been extensively used to identify genes involved in cortical migration. In all prior cases-JNK, Rac1, STEF/Tiam1, p27, LIS1, Ndel1, cytoplasmic dynein, doublecortin, doublecortin-like kinase, MDGA1, DYX1C1, KIAA0319, and Dab1 (Kawauchi et al. 2003, 2006; Shu et al. 2004; Tsai et al. 2005; Koizumi et al. 2006; Olson et al. 2006; Paracchini et al. 2006; Takeuchi and O'Leary 2006; Wang et al. 2006)—inhibiting function inhibits migration and causes neurons to settle more deeply in or below the cortical plate. However, to our knowledge, the phenotype we observe in E14.5 neurons expressing Cul5 shRNA is unprecedented, 

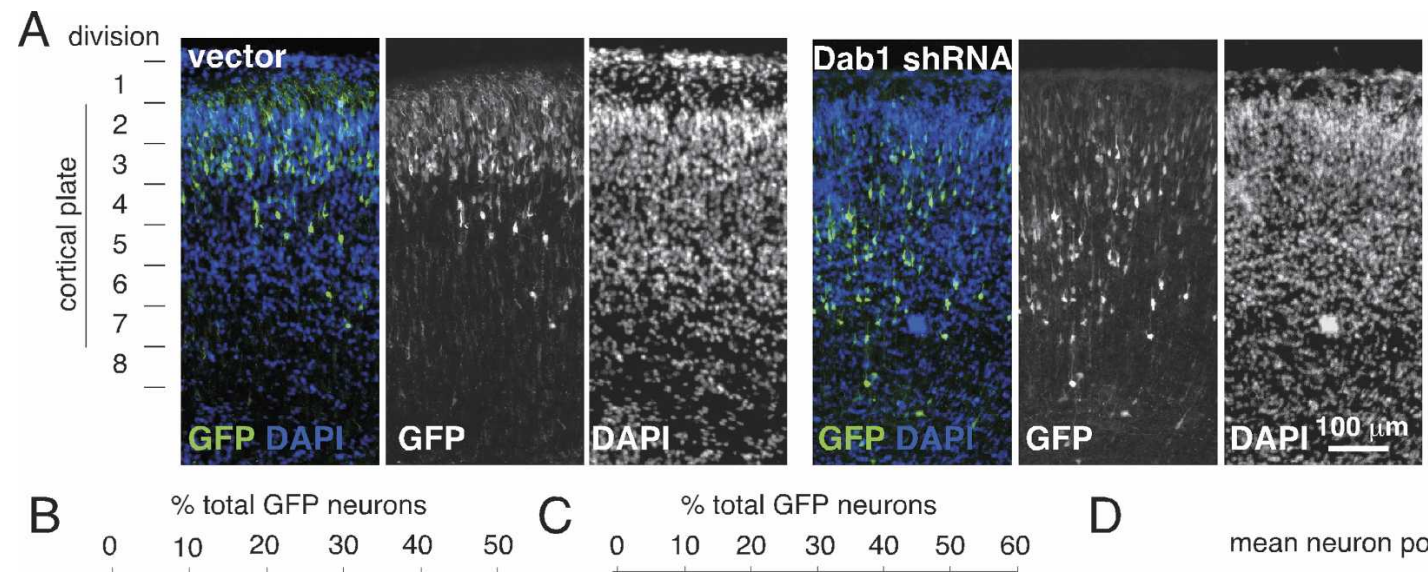

D
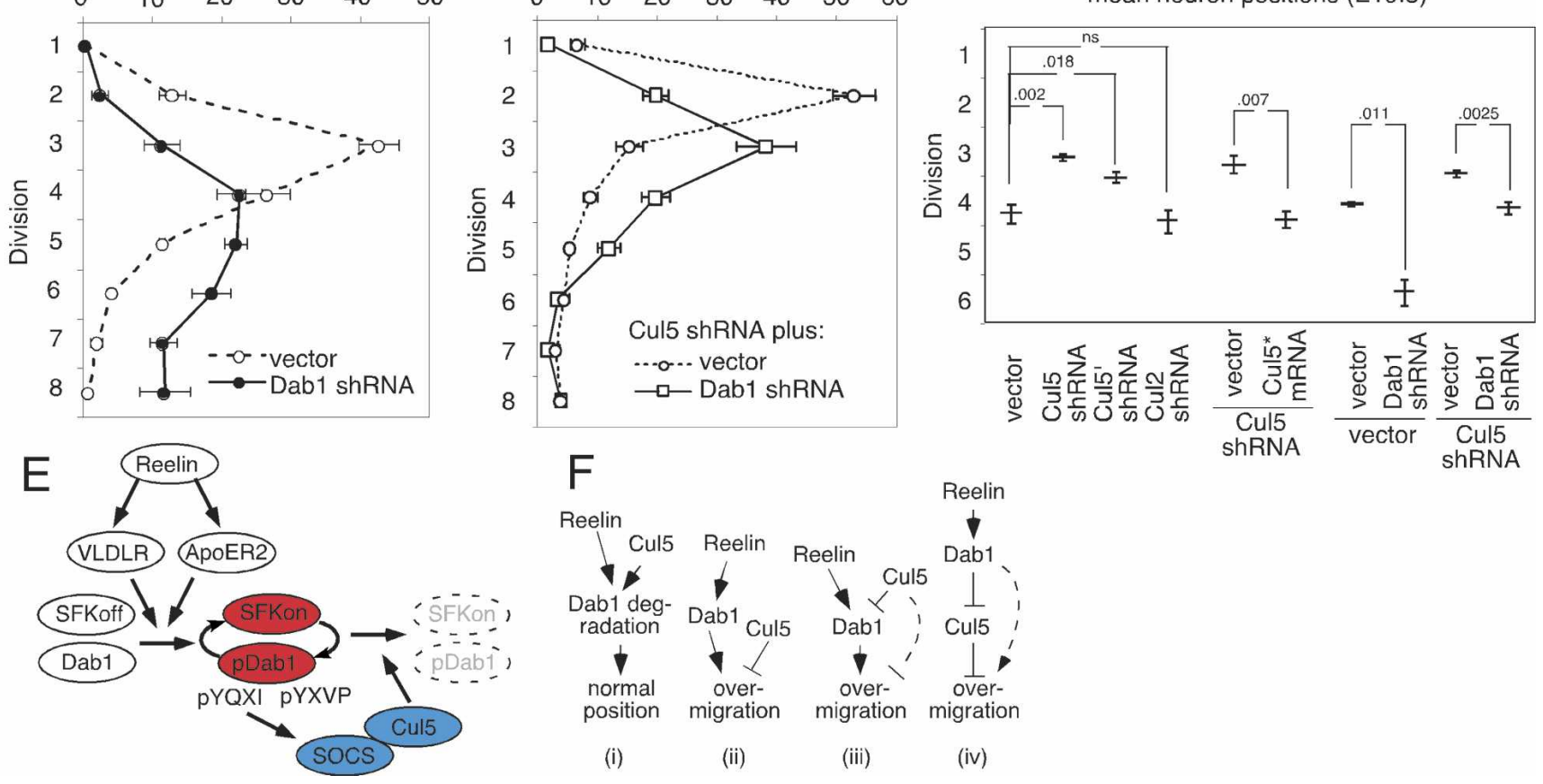

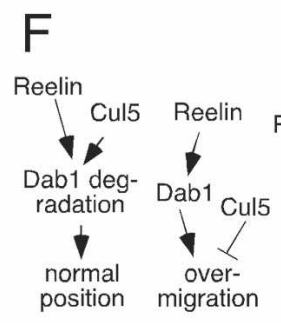

(i)

(ii)

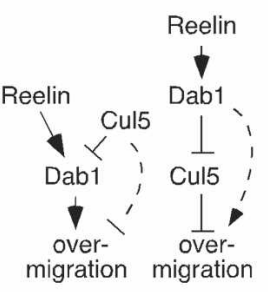

(iii)

(iv)

Figure 8. Relationship between Cul5 and Dab1 effects on neuron positioning. (A) Positions of GFP-positive neurons at E19.5, after in utero microinjection at E14.5 with $2 \mu \mathrm{g}$ of vector or Dab1 shRNA and $0.5 \mu \mathrm{g}$ of GFP plasmid pCAGIG. Cortical divisions used for quantification are indicated on the left. $(B)$ Quantification of neuron positions at E19.5. Mean \pm SEM for $n=2$ vector and $n=4$ Dab1 shRNA embryos. Average neurons were vector, $3.58 \pm 0.05$; and Dab1 shRNA, $5.37 \pm 0.26(P=0.011)$. (C) Combined Dab1 and Cul5 shRNAs. Embryos were injected with $0.5 \mu \mathrm{g}$ of Cul 5 shRNA and $0.5 \mu \mathrm{g}$ of pCAGIG, together with $2 \mu \mathrm{g}$ of either vector or Dab1 shRNA, and analyzed at E19.5. Mean \pm SEM for $n=7$ vector and $n=6$ Dab1 shRNA embryos. Average neuron positions were Cul5 shRNA plus vector, $2.97 \pm 0.08$; and Cul5 shRNA plus Dab1 shRNA, $3.66 \pm 0.12(P=0.0025)$. $(D)$ Summary of mean neuron positions caused by electroporation of different constructs. Mean \pm SEM and $P$ values for each matched set of injections. The gray region shows normal neuron positions for each of the controls. (ns) Not significant. (E) Molecular mechanism. Reelin binds to partially redundant receptors, VLDLR and ApoER2, to induce activation of SFKs (Src and Fyn) and tyrosine phosphorylation of Dab1, in a positive feedback loop. SOCS and Cul5 target phosphorylated Dab1, and possibly SFKs, for ubiquitylation and degradation, down-regulating signaling in a negative feedback loop. $(F)$ Contrasting models for the relationship between Cul5, Dab1, and neuron position. (Model i) It was possible that lack of Reelin-induced Dab1 down-regulation was the cause of the Reeler phenotype. However, the effect of blocking Dab1 down-regulation, with Cul5 shRNA, is clearly different from blocking Reelin signaling, by Dab1 shRNA or in Reeler or Dab1 mutants (shown in B), so this model is unlikely to be correct. (Model ii) Cul5 may inhibit migration and Dab1 may stimulate migration, through independent pathways. (Model iii) Cul5 lowers the level of Reelin-activated Dab1, which promotes neuron migration. The lack of epistasis between Dab1 and Cul5 (Fig. 7C) suggests that Cul5 may have other important targets that also regulate neuron migration. (Model iv) Activated Dab1 inhibits Cul5, which inhibits neuron migration. The lack of epistasis between Dab1 and Cul5 suggests that Dab1 may have Cul5-independent mechanisms to promote migration. Our results do not distinguish models ii-iv.

with electroporated neurons accumulating at the top of the cortical plate. The neurons at the boundary of the marginal zone have short, branched ascending processes and cell bodies that are close to but do not pass beyond the Cajal-Retzius neurons. The cells appear to be neurons because they extend axons into the subplate/inter- 
mediate zone region and toward the contralateral hemisphere. They express neuronal markers appropriate for their time of origin. Thus, like Reelin or Dab1 mutations, depletion of Cul5 appears to affect cell position but not fate.

Full understanding of the cellular basis for this phenotype will require more detailed live imaging studies. However, we can use our data from two time points after electroporation to construct the likely migratory path when Cul5 is depleted. The Cul5 shRNA neurons appear to migrate at normal or slightly increased speed to the top of the cortical plate and then are displaced outward, apparently because subsequent neurons are unable to pass them. We call this an "overmigration" phenotype. This is the "opposite" of the path followed by Dab1 shRNA neurons, which migrate at a slower rate than controls and settle below their normal position (Fig. 8B; Olson et al. 2006). As mentioned above, no other publication using shRNA to study neuronal migration has reported overmigration, so off-target effects are considered unlikely. In addition, the phenotype was seen with two different Cul5 shRNAs, was not induced by vector, Cul2 or Dab1 shRNA electroporation, and was rescued by coexpressing Cul5 protein. Therefore, we consider that Cul5 has a unique role in preventing overmigration of neurons when they reach the top of the cortical plate.

A causal link between Dabl activation and the cellular migration defect remains to be established. Prior to our experiments, there were several possibilities, illustrated in Figure 8F. The first possibility (Fig. 8F, model i), raised originally by Rice et al. (1998), is that accumulation of Dab1 protein-i.e., absence of Reelin-induced Dab1 degradation-causes the Reeler phenotype. This hypothesis is consistent with the finding that the Reeler mutant phenotype, whether caused by mutations in Reelin, the receptors, or SFKs, correlates with buildup of Dab1. If this scenario were true, inhibiting the Dab1 degradation machinery (by Cul5 shRNA) might cause the same phenotype as absence of Reelin or Dab1. However, inhibiting down-regulation of phosphorylated Dab1 causes a phenotype that appears to be the converse of that caused when Dab1 is absent. Thus the results argue against a positive role for Dab1 degradation in the Reelin signaling.

Because Dab1 and Cul5 have opposing effects (Fig. 8), there are three possibilities for how Dab1 and Cul5 may interact: They may be independent; Cul5 may inhibit Dab1, which promotes neuron migration; or Dab1 may inhibit Cul5, which inhibits neuron migration (Fig. 8F, panels ii-iv, respectively). Such possibilities are generally distinguished by epistasis. We found that neither Dab1 shRNA nor Cul5 shRNA was epistatic to the other (Fig. 8C). This suggests that models iii and iv need to be modified by the addition of indirect pathways (Fig. 8, broken lines). For example, Cul5 may have other targets besides Dab1 that promote neuron migration (model iii), or Dab1 may have other targets besides Cul5 that inhibit neuron migration (model iv). Alternatively, our epistasis results may be misleading. While control experiments showed that new Dab1 protein synthesis is inhibited when both Dab1 and Cul5 shRNAs are present (Supplementary Fig. S8), nonphosphorylated Dab1 protein is very stable and may not be removed by shRNA in vivo. This Dab1 protein may be phosphorylated in response to Reelin and have a longer half-life when Cu15 is absent. True epistasis testing will require the use of Dab1 and Cul5 mutants.

Although we cannot exclude models ii and iv, we favor model iii because Reelin was previously proposed to stimulate neuronal movement to the top of the cortical plate by facilitating a process called somal translocation (Nadarajah et al. 2001). Somal translocation requires detachment of neurons from their radial glia guides, which is stimulated by Reelin (Dulabon et al. 2000). In addition, our results suggest that Reelin-Dabl signaling may actively promote somal translocation until such time as active Dab1 is degraded by Cul5. Thus Reelin is a "detach and go" signal. Dab1 down-regulation would be the "stop" signal that allows the neuron to stop moving and be passed by its younger sisters.

The exact mechanism of Cul5 action during lamination of the cortex remains to be explored by a conventional mutation approach. We only tested the effect of Cul5 knockdown at a particular time of development, and we cannot predict how migrations of earlier or later born cortical neurons might be affected. However, it seems likely that Cul5 mutation will affect cortical development, perhaps causing a novel layering phenotype that would be ameliorated by Reelin or Dab1 mutation.

Cul5 may also regulate other Reelin-dependent developmental events, including neuron migrations in the hippocampus, cerebellum, olfactory lobe, hindbrain, and spinal cord, and dendrite outgrowth in the hippocampus (Rice and Curran 2001; Gupta et al. 2002; Jossin 2004; Herz and Chen 2006). Cul5 may also affect nondevelopmental roles of Reelin, such as synaptic modulation, learning, and memory (Herz and Chen 2006). If SOCSCul5 complexes are more widely involved in down-regulating other aspects of Src family kinase function, Cul5 knockout may affect additional aspects of brain development and function.

\section{Materials and methods}

\section{Antibodies}

The following antibodies were used for biochemistry: mouse anti-phosphotyrosine (4G10) (Upstate Biotechnology); rabbit anti-Fyn (FYN3), anti-Dab1 (E19), anti-SOCS1 (H-93), and antiCul5 (H-300) (Santa Cruz Biotechnology); mouse anti-Flag (M2) (Sigma); mouse anti-GFP (Roche); mouse anti-HA.11 and antiTuj1 (Covance); mouse anti-BrdU and Dab2 (Becton Dickinson); chicken anti-GFP (Ab16901) (Chemicon); mouse anti-myc (9E10) (BAbCO); and affinity-purified rabbit anti-Dab1 (B3) from Brian Howell. For immunofluorescence, we used rabbit antiGFP (A11122) (Molecular Probes), mixed monoclonal anti-GFP (Roche), rabbit anti-Dab1 (B3), goat anti-Dab1 C terminus (Exalpha), rabbit Dab1pY220 (Biosource), hamster anti-Brn1 (Robert McEvilly), rabbit anti-Tbr1 (Robert Hevner), and donkey secondary antibodies labeled with Alexa488 and 568 (Molecular Probes). 


\section{Cell culture and transfection}

COS7 and HeLa cells were maintained in Dulbecco's modified Eagle's medium (DMEM) supplemented with 10\% FBS, $100 \mathrm{IU} /$ $\mathrm{mL}$ penicillin, and $100 \mu \mathrm{g} / \mathrm{mL}$ streptomycin. COS and HeLa cell transfections were performed using Lipofectamine Plus according to the manufacturer's instructions. Expression vectors are described in the Supplemental Material. After $24 \mathrm{~h}$ of transfection, cells were lysed and subjected to Western blotting and immunoprecipitation as described previously (Arnaud et al. 2003a; Ballif et al. 2003). Unsaturated enhanced chemiluminescence images were scanned into Adobe Photoshop and quantified using ImageJ.

\section{Neuron cultures and electroporations}

Neuron suspensions were prepared from E16.5 mouse embryo cerebral cortex and electroporated essentially as described previously (Herrick and Cooper 2002; Xu et al. 2005). The vectors are described in the Supplemental Material. Equal cell numbers from each electroporation were plated in two precoated $35-\mathrm{mm}$ dishes. For acute stimulation with Reelin, cultures were incubated for 4-5 d and then stimulated for 15 min with Reelin that had been harvested in Neurobasal medium from $293 \mathrm{~T}$ cells stably transfected with a Reelin expression plasmid (Ballif et al. 2003). The control plate was treated with medium harvested similarly from 293T cells stably transfected with vector DNA. For long-term treatment, cultures were treated either with Reelin-containing medium or with control medium containing the extracellular domain of VLDLR $\left(20 \mu \mathrm{g} / \mathrm{mL} \mathrm{MBPV}_{1-8} \mathrm{His}\right)$ (Koch et al. 2002) to sequester endogenous Reelin. Treatment started $2 \mathrm{~d}$ after plating and continued for $4 \mathrm{~d}$. Neurons were washed and lysed in NP40 buffer (Ballif et al. 2003), and protein concentrations were equalized.

\section{$\operatorname{shRNA}$}

Cul2, Cul5, Cul5', GFP shRNAs, and pMXpuro empty vector were kindly provided by Drs. Takumi Kamura and Keiichi Nakayama, Kyushu University, Fukuoka, Japan (Kamura et al. 2004). The target sequences are Cul2, GAGCTAGCATTGGATATG TGG; Cul5, GCTGCAGACTGAATTAGTAG; and Cul5', GTC TCACTTCCTACTGAACTG. pCAGIG Cul5 wt and pCAGIG Cul5* were constructed by moving wild-type human myc-Cul5 from pcDNA3 (kind gift of J. Wade Harper) into pCAGIG using PCR and 5' XhoI and 3' NotI sites. We then introduced five silent mutations into the sequence targeted by Cul5 shRNA (GCTGCAGACAGAGCTCGTGG). Dab1 shRNA hairpin vectors $\mathrm{A}$ and $\mathrm{B}$ were built by inserting annealed oligos with the target sequences AATCACAGATTTGTGGCCATC (Olson et al. 2006) and AGCCGCCTTCATGCCCACACA into the BglII and HindIII restriction sites in the pSuper_retro_puro vector.

\section{In utero microinjection and electroporation}

Timed pregnant CD-1 mice were obtained from Charles River Laboratories and manipulated according to Tabata and Nakajima (2001) at E14.5. Needles for injection were pulled from Wiretrol II glass capillaries (Drummond Scientific) and calibrated for $1-\mu \mathrm{L}$ injections with the supplied wire plunger. DNA solutions were mixed to final concentration of $2 \mathrm{mg} / \mathrm{mL}$ in 10 $\mathrm{mM}$ Tris $(\mathrm{pH} 8.0)$ with $0.01 \%$ Fast Green. Forceps-type electrodes (Nepagene) with $3-\mathrm{mm}$ pads were used for electroporation (five $50-\mathrm{msec}$ pulses of $45 \mathrm{~V}$ ).

\section{Histology}

Embryos were recovered at either E17.5 or E19.5 (P0), and brains were dissected using a microscope equipped for epifluorescence.
GFP-positive brains were fixed in a $4 \%$ paraformaldehyde, $4 \%$ sucrose, PBS solution for $4 \mathrm{~h}$ at room temperature. This was removed and replaced with $10 \%$ sucrose/PBS overnight, then $30 \%$ sucrose for $1 \mathrm{~d}$ before freezing in O.T.C. compound. Twenty-micrometer-thick brain cross-sections were obtained with a cryostat.

Sections were permeabilized in TBS with $0.1 \%$ Tween 20 for 20 min and blocked for $2 \mathrm{~h}$ with 3\% BSA, 5\% calf serum, and $0.1 \%$ Triton X-100 in PBS. Primary antibodies were incubated overnight at $4^{\circ} \mathrm{C}$. Slides were washed twice for $10 \mathrm{~min}$ in $0.1 \%$ Tween 20/TBS. Secondary antibodies were added for $1 \mathrm{~h}$ at room temperature. Slides were washed as before, rinsed with $\mathrm{ddH}_{2} \mathrm{O}$, and coverslipped with Prolong Gold anti-fade reagent (Molecular Probes). Most images were obtained with a $10 \times$ objective and captured with Metamorph software. High-magnification images used a $40 \times$ oil objective and were processed using Deltavision deconvolution software. Images were assembled in Adobe Photoshop and Deneba Canvas. Contrast $(\gamma)$ was not adjusted.

We quantified neuron position as described in Results, counting 100-400 $\mathrm{GFP}^{+}$neurons from several sections per embryo. Medial (cingulate) electroporations were not scored. Data are mostly from the lateral part of the anterior neocortex (Supplementary Fig. S6A). The mean neuron position $\left(D_{\mathrm{m}}\right)$ in each embryo was calculated as $\Sigma\left(N_{i} D_{i}\right) / \Sigma\left(N_{i}\right)$, where $N_{i}$ is the number of $\mathrm{GFP}^{+}$neurons in division $D_{i}$. The mean and standard error of $D_{\mathrm{m}}$ were then calculated for $n$ embryos. $P$ values are by Student's $t$-test, two tailed, unequal variance.

\section{Acknowledgments}

We are very grateful to Susan Veals, Priscilla Kronstadt O'Brien, and Sandra Chang for trouble-shooting in utero electroporation methods, and to Kamon Sanada, Yasu Ito, and Yukiko Gotoh for their suggestions. Priscilla Kronstadt and Jake Goodchild provided valuable technical assistance. We greatly appreciate the prompt gifts of Cullin and SOCS reagents from Keichii Nakayama, Chris Greenhalgh, Neil Nathanson, and Wade Harper. Many thanks also to Lionel Arnaud, Brian Howell, C. Jane McGlade, Ze'ev Ronai, Stanley Lipkowitz, Kamon Sanada, Stephanie Kaech, Tohru Tezuka, Johannes Nimpf, Robert McEvilly, Robert Hevner, Valeri Vasioukhin, and Yasu Fujita for reagents, and to Robert Hevner and Cecilia Moens for thoughtful comments on the manuscript. This work was supported by grant CA41072 from the National Cancer Institute.

\section{References}

Arnaud, L., Ballif, B.A., and Cooper, J.A. 2003a. Regulation of protein tyrosine kinase signaling by substrate degradation during brain development. Mol. Cell. Biol. 23: 9293-9302.

Arnaud, L., Ballif, B.A., Forster, E., and Cooper, J.A. 2003b. Fyn tyrosine kinase is a critical regulator of Disabled-1 during brain development. Curr. Biol. 13: 9-17.

Ballif, B.A., Arnaud, L., and Cooper, J.A. 2003. Tyrosine phosphorylation of disabled-1 is essential for Reelin-stimulated activation of Akt and Src family kinases. Brain Res. Mol. Brain Res. 117: 152-159.

Ballif, B.A., Arnaud, L., Arthur, W.T., Guris, D., Imamoto, A., and Cooper, J.A. 2004. Activation of a Dab1/CrkL/C3G/ Rap1 pathway in Reelin-stimulated neurons. Curr. Biol. 14: 606-610.

Bock, H.H. and Herz, J. 2003. Reelin activates SRC family tyrosine kinases in neurons. Curr. Biol. 13: 18-26.

Bock, H.H., Jossin, Y., May, P., Bergner, O., and Herz, J. 2004. 
Apolipoprotein E receptors are required for reelin-induced proteasomal degradation of the neuronal adaptor protein Disabled-1. J. Biol. Chem. 279: 33471-33479.

De Sepulveda, P., Okkenhaug, K., Rose, J.L., Hawley, R.G., Dubreuil, P., and Rottapel, R. 1999. Socs1 binds to multiple signalling proteins and suppresses steel factor-dependent proliferation. EMBO J. 18: 904-915.

De Souza, D., Fabri, L.J., Nash, A., Hilton, D.J., Nicola, N.A., and Baca, M. 2002. SH2 domains from suppressor of cytokine signaling-3 and protein tyrosine phosphatase SHP-2 have similar binding specificities. Biochemistry 41: 9229-9236.

Dulabon, L., Olson, E.C., Taglienti, M.G., Eisenhuth, S., McGrath, B., Walsh, C.A., Kreidberg, J.A., and Anton, E.S. 2000. Reelin binds $\alpha 3 \beta 1$ integrin and inhibits neuronal migration. Neuron 27: 33-44.

Ferrell Jr., J.E. 1996. Tripping the switch fantastic: How a protein kinase cascade can convert graded inputs into switchlike outputs. Trends Biochem. Sci. 21: 460-466.

Gupta, A., Tsai, L.H., and Wynshaw-Boris, A. 2002. Life is a journey: A genetic look at neocortical development. Nat. Rev. Genet. 3: 342-355.

Hammond, V., Howell, B., Godinho, L., and Tan, S.S. 2001. disabled-1 functions cell autonomously during radial migration and cortical layering of pyramidal neurons. J. Neurosci. 21: 8798-8808.

Hatten, M.E. 2002. New directions in neuronal migration. Science 297: 1660-1663.

Heinrich, R., Neel, B.G., and Rapoport, T.A. 2002. Mathematical models of protein kinase signal transduction. Mol. Cell 9: 957-970.

Herrick, T.M. and Cooper, J.A. 2002. A hypomorphic allele of dab1 reveals regional differences in reelin-Dab1 signaling during brain development. Development 129: 787-796.

Herz, J. and Chen, Y. 2006. Reelin, lipoprotein receptors and synaptic plasticity. Nat. Rev. Neurosci. 7: 850-859.

Hilton, D.J., Richardson, R.T., Alexander, W.S., Viney, E.M., Willson, T.A., Sprigg, N.S., Starr, R., Nicholson, S.E., Metcalf, D., and Nicola, N.A. 1998. Twenty proteins containing a C-terminal SOCS box form five structural classes. Proc. Natl. Acad. Sci. 95: 114-119.

Hirano, T. and Murakami, M. 2006. Grasp a pTyr-peptide by its SOCS. Dev. Cell 10: 542-544.

Howell, B.W., Herrick, T.M., Hildebrand, J.D., Zhang, Y., and Cooper, J.A. 2000. Dab1 tyrosine phosphorylation sites relay positional signals during mouse brain development. Curr. Biol. 10: 877-885.

Ilangumaran, S., Ramanathan, S., and Rottapel, R. 2004. Regulation of the immune system by SOCS family adaptor proteins. Semin. Immunol. 16: 351-365.

Jossin, Y. 2004. Neuronal migration and the role of reelin during early development of the cerebral cortex. Mol. Neurobiol. 30: 225-251.

Kamizono, S., Hanada, T., Yasukawa, H., Minoguchi, S., Kato, R., Minoguchi, M., Hattori, K., Hatakeyama, S., Yada, M., Morita, S., et al. 2001. The SOCS box of SOCS-1 accelerates ubiquitin-dependent proteolysis of TEL-JAK2. J. Biol. Chem. 276: $12530-12538$.

Kamura, T., Maenaka, K., Kotoshiba, S., Matsumoto, M., Kohda, D., Conaway, R.C., Conaway, J.W., and Nakayama, K.I. 2004. VHL-box and SOCS-box domains determine binding specificity for Cul2-Rbx1 and Cul5-Rbx2 modules of ubiquitin ligases. Genes \& Dev. 18: 3055-3065.

Kawauchi, T., Chihama, K., Nabeshima, Y., and Hoshino, M. 2003. The in vivo roles of STEF/Tiam1, Racl and JNK in cortical neuronal migration. EMBO J. 22: 4190-4201.

Kawauchi, T., Chihama, K., Nabeshima, Y.I., and Hoshino, M.
2006. Cdk5 phosphorylates and stabilizes p27(kip1) contributing to actin organization and cortical neuronal migration. Nat. Cell Biol. 8: 17-26.

Keshvara, L., Benhayon, D., Magdaleno, S., and Curran, T. 2001. Identification of reelin-induced sites of tyrosyl phosphorylation on disabled 1. J. Biol. Chem. 276: 16008-16014.

Koch, S., Strasser, V., Hauser, C., Fasching, D., Brandes, C., Bajari, T.M., Schneider, W.J., and Nimpf, J. 2002. A secreted soluble form of ApoE receptor 2 acts as a dominant-negative receptor and inhibits Reelin signaling. EMBO J. 21: 59966004.

Koizumi, H., Tanaka, T., and Gleeson, J.G. 2006. Doublecortinlike kinase functions with Doublecortin to mediate fiber tract decussation and neuronal migration. Neuron 49: 55-66.

Krebs, D.L. and Hilton, D.J. 2000. SOCS: Physiological suppressors of cytokine signaling. I. Cell Sci. 113: 2813-2819.

Krebs, D.L., Metcalf, D., Merson, T.D., Voss, A.K., Thomas, T., Zhang, J.G., Rakar, S., O'Bryan, M.K., Willson, T.A., Viney, E.M., et al. 2004. Development of hydrocephalus in mice lacking SOCS7. Proc. Natl. Acad. Sci. 101: 15446-15451.

Kriegstein, A.R. and Noctor, S.C. 2004. Patterns of neuronal migration in the embryonic cortex. Trends Neurosci. 27: 392-399.

Kriegstein, A., Noctor, S., and Martinez-Cerdeno, V. 2006. Patterns of neural stem and progenitor cell division may underlie evolutionary cortical expansion. Nat. Rev. Neurosci. 7: 883-890.

Kuo, G., Arnaud, L., Kronstad-O'Brien, P., and Cooper, J.A. 2005. Absence of Fyn and Src causes a reeler-like phenotype. J. Neurosci. 25: 8578-8586.

Liu, E., Cote, J.F., and Vuori, K. 2003. Negative regulation of FAK signaling by SOCS proteins. EMBO J. 22: 5036-5046.

Marin, O. and Rubenstein, J.L. 2003. Cell migration in the forebrain. Annu. Rev. Neurosci. 26: 441-483.

Ming, G.L., Wong, S.T., Henley, J., Yuan, X.B., Song, H.J., Spitzer, N.C., and Poo, M.M. 2002. Adaptation in the chemotactic guidance of nerve growth cones. Nature 417: 411418.

Nadarajah, B., Brunstrom, J.E., Grutzendler, J., Wong, R.O., and Parnavelas, J.G. 2001. Two modes of neuronal migration in early development of the cerebral cortex. Nat. Neurosci. 4: 143-150.

Nadarajah, B., Alifragis, P., Wong, R.O., and Parnavelas, J.G. 2003. Neuronal migration in the developing cerebral cortex: Observations based on real-time imaging. Cereb. Cortex 13: 607-611.

Olson, E.C., Kim, S., and Walsh, C.A. 2006. Impaired neuronal positioning and dendritogenesis in the neocortex after cellautonomous Dab1 suppression. J. Neurosci. 26: 1767-1775.

Paracchini, S., Thomas, A., Castro, S., Lai, C., Paramasivam, M., Wang, Y., Keating, B.J., Taylor, J.M., Hacking, D.F., Scerri, T., et al. 2006. The chromosome 6p22 haplotype associated with dyslexia reduces the expression of KIAA0319, a novel gene involved in neuronal migration. Hum. Mol. Genet. 15: 1659-1666.

Park, T.J., Hamanaka, H., Ohshima, T., Watanabe, N., Mikoshiba, K., and Nukina, N. 2003. Inhibition of ubiquitin ligase Siah-1A by disabled-1. Biochem. Biophys. Res. Commun. 302: 671-678.

Petroski, M.D. and Deshaies, R.J. 2005. Function and regulation of cullin-RING ubiquitin ligases. Nat. Rev. Mol. Cell Biol. 6: 9-20.

Polizzotto, M.N., Bartlett, P.F., and Turnley, A.M. 2000. Expression of 'suppressor of cytokine signalling' (SOCS) genes in the developing and adult mouse nervous system. J. Comp. Neurol. 423: 348-358. 
Feng et al.

Rakic, P. 1995. A small step for the cell, a giant leap for mankind: A hypothesis of neocortical expansion during evolution. Trends Neurosci. 18: 383-388.

Rice, D.S. and Curran, T. 2001. Role of the reelin signaling pathway in central nervous system development. Annu. Rev. Neurosci. 24: 1005-1039.

Rice, D.S., Sheldon, M., D'Arcangelo, G., Nakajima, K., Goldowitz, D., and Curran, T. 1998. Disabled-1 acts downstream of Reelin in a signaling pathway that controls laminar organization in the mammalian brain. Development 125: 37193729.

Sanada, K., Gupta, A., and Tsai, L.H. 2004. Disabled-1-regulated adhesion of migrating neurons to radial glial fiber contributes to neuronal positioning during early corticogenesis. Neuron 42: 197-211.

Shu, T., Ayala, R., Nguyen, M.D., Xie, Z., Gleeson, J.G., and Tsai, L.H. 2004. Ndell operates in a common pathway with LIS1 and cytoplasmic dynein to regulate cortical neuronal positioning. Neuron 44: 263-277.

Suetsugu, S., Tezuka, T., Morimura, T., Hattori, M., Mikoshiba, K., Yamamoto, T., and Takenawa, T. 2004. Regulation of actin cytoskeleton by mDab1 through N-WASP and ubiquitination of mDab1. Biochem. J. 384: 1-8.

Tabata, H. and Nakajima, K. 2001. Efficient in utero gene transfer system to the developing mouse brain using electroporation: Visualization of neuronal migration in the developing cortex. Neuroscience 103: 865-872.

Tabata, H. and Nakajima, K. 2002. Neurons tend to stop migration and differentiate along the cortical internal plexiform zones in the Reelin signal-deficient mice. J. Neurosci. Res. 69: 723-730.

Takeuchi, A. and O'Leary, D.D. 2006. Radial migration of superficial layer cortical neurons controlled by novel Ig cell adhesion molecule MDGA1. J. Neurosci. 26: 4460-4464.

Trommsdorff, M., Gotthardt, M., Hiesberger, T., Shelton, J., Stockinger, W., Nimpf, J., Hammer, R.E., Richardson, J.A., and Herz, J. 1999. Reeler/Disabled-like disruption of neuronal migration in knockout mice lacking the VLDL receptor and ApoE receptor 2. Cell 97: 689-701.

Tsai, J.W., Chen, Y., Kriegstein, A.R., and Vallee, R.B. 2005. LIS1 RNA interference blocks neural stem cell division, morphogenesis, and motility at multiple stages. J. Cell Biol. 170: 935-945.

Turnley, A.M., Faux, C.H., Rietze, R.L., Coonan, J.R., and Bartlett, P.F. 2002. Suppressor of cytokine signaling 2 regulates neuronal differentiation by inhibiting growth hormone signaling. Nat. Neurosci. 5: 1155-1162.

Wang, Y., Paramasivam, M., Thomas, A., Bai, J., KaminenAhola, N., Kere, J., Voskuil, J., Rosen, G.D., Galaburda, A.M., and Loturco, J.J. 2006. DYX1C1 functions in neuronal migration in developing neocortex. Neuroscience 143: 515522.

Weiner, O.D. 2002. Regulation of cell polarity during eukaryotic chemotaxis: The chemotactic compass. Curr. Opin. Cell Biol. 14: 196-202.

Xu, M., Arnaud, L., and Cooper, J.A. 2005. Both the phosphoinositide and receptor binding activities of Dabl are required for Reelin-stimulated Dab1 tyrosine phosphorylation. Brain Res. Mol. Brain Res. 139: 300-305.

Yi, T.M., Huang, Y., Simon, M.I., and Doyle, J. 2000. Robust perfect adaptation in bacterial chemotaxis through integral feedback control. Proc. Natl. Acad. Sci. 97: 4649-4653. 


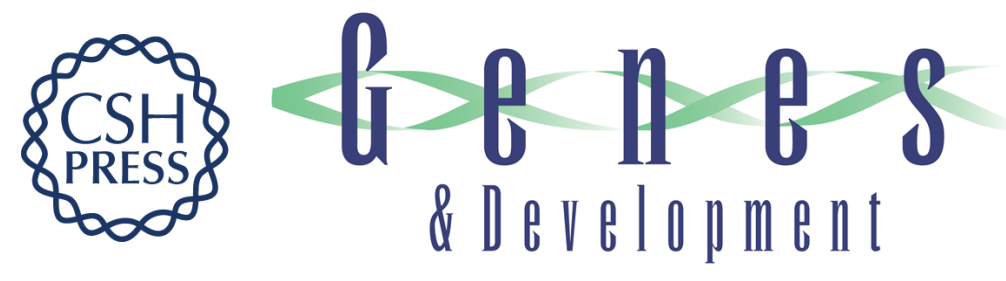

\section{Cullin 5 regulates Dab1 protein levels and neuron positioning during cortical development}

Libing Feng, Nathaniel S. Allen, Sergi Simo, et al.

Genes Dev. 2007, 21:

Access the most recent version at doi:10.1101/gad.1604207

\section{Supplemental http://genesdev.cshlp.org/content/suppl/2007/10/18/21.21.2717.DC1 \\ Material}

Related Content A missed exit: Reelin sets in motion Dab1 polyubiquitination to put the break on neuronal migration

Géraldine Kerjan and Joseph G. Gleeson

Genes Dev. November , 2007 21:2850-2854

References This article cites 59 articles, 20 of which can be accessed free at: http://genesdev.cshlp.org/content/21/21/2717.full.html\#ref-list-1

Articles cited in:

http://genesdev.cshlp.org/content/21/21/2717.full.html\#related-urls

License Freely available online through the Genes \& Development Open Access option.

$\begin{array}{cl}\text { Email Alerting } & \begin{array}{l}\text { Receive free email alerts when new articles cite this article - sign up in the box at the top } \\ \text { right corner of the article or click here. }\end{array}\end{array}$

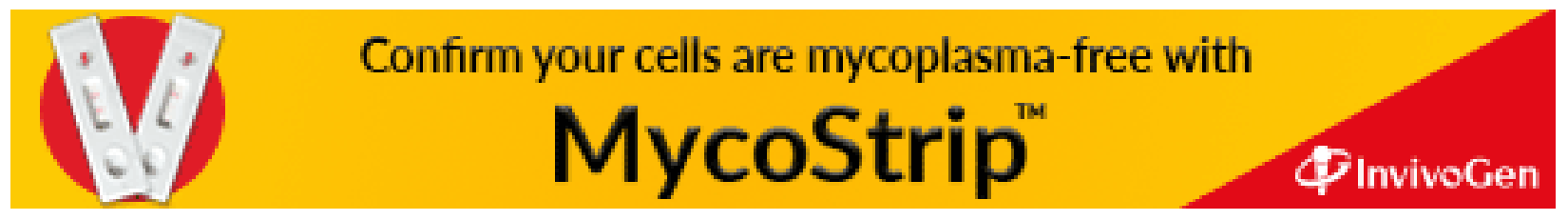

